\title{
Insulin Resistance Modulation with Lifestyle Modification- Proof-of-Concept Study in Rats
}

\author{
Afzal Haq Asif ${ }^{1, \star}$, Prem Kumar Nanjundan², Girish Meravanige Basavarajappa ${ }^{3}$, Nagaraja \\ Sreeharsha ${ }^{4,5, \star}$ Uma Chandur ${ }^{6}$ and Teeka Srinivasasetty Roopashree ${ }^{7}$
}

1'Department of Pharmacy Practice, College of Clinical Pharmacy, King Faisal University, Al-Ahsa, SAUDI ARABIA. ${ }^{2}$ Department of Pharmacology, Vidya Siri College of Pharmacy, Off Sarjapura Road, Bangalore, Karnataka, INDIA. ${ }^{3}$ Department of Biomedical Sciences, College of Medicine, King Faisal University, Al-Ahsa, SAUDI ARABIA.

${ }^{4}$ Department of Pharmaceutical Sciences, College of Clinical Pharmacy, King Faisal University, Al-Ahsa, SAUDI ARABIA. ${ }^{5}$ Department of Pharmaceutics, Vidya Siri College of Pharmacy, Off Sarjapura Road, Bangalore, Karnataka, INDIA. ${ }^{6}$ Department of Pharmacognosy, Vidya Siri College of Pharmacy, Off Sarjapura Road, Bangalore, Karnataka, INDIA. ${ }^{7}$ Department of Pharmacognosy, Government College of Pharmacy, Bangalore, Karnataka, INDIA.

\begin{abstract}
Purpose: Fatigue, a physical and/or mental exhaustion is a common symptom of diabetes. Easy fatigability is not limited to uncontrolled diabetes however is comorbid with psychological, medical, metabolic, and chronic complications like insulin-resistance, glucose intolerance, and obesity. Methods: We analyzed the effect of lifestyle modification involving physical exercise and intake of omega 3 fatty acid-rich fish oil on insulin resistance and diabetic fatigue. For the same, diabetes was induced using single dose streptozocin injection in rats fed with a high-fat diet and were treated with fish oil alone and in combination with exercise training. Blood glucose levels, glucose tolerance, lipid profile, insulin levels, oxidative stress and glycogen content were monitored in rats exposed to swimming exercise and fatigue test. Results: Alone fish oil could improve symptoms of diabetic complications; however, the combination intervention of exercise and oral administration of fish oil significantly lowered the fasting blood glucose level and fatty acid profile in diabetic rats. Improvement in antioxidant profile was observed from the decreased levels of lipid peroxidation products and increased levels of antioxidants like superoxide dismutase and glutathione. Effective glucose disposal correlated with better insulin tolerance and improvement in lipid profile as indicated by a significant reduction in LDL, VLDL, free fatty acids, and leptin with an increase in HDL levels. Exercise-induced changes in the liver and skeletal tissue, enabling diabetic rats to restore their glycogen stores thus building the endurance required for prolonged exercise. Conclusion: Lifestyle modification with moderate exercise and consumption of antioxidant-rich fish oil is a novel and easy therapeutic intervention in the treatment of multifactorial syndrome of fatigue observed as a diabetic complication.
\end{abstract}

Key words: Insulin, Fatigue, Diabetes, Fish Oil, Exercise.

\section{INTRODUCTION}

Insulin resistance is approaching epidemic proportions globally due to a sedentary lifestyle accompanied by a diet with a high content of carbohydrate and transfat. ${ }^{1}$ Fatigue is distinctly associated with diabetes mellitus and is directly correlated with the severity of the disease and complications. ${ }^{2}$ The clinical symptoms of type 2 diabetes are increased blood glucose concentration, oxidative stress, insulin resistance, hyperleptinemia, hypertension, and fatigue. Fatigue occurs very frequently in diabetic patients and it indicates the severity of hyperglycemic status and its complications. ${ }^{3-5}$ Oxidative stress and insulin resistance are directly corelated. ${ }^{6}$ Excess production of reactive free radicals is due to abnormal metabolism of lipids and glucose. High-fat consumption further adds a burden to the antioxidative system. ${ }^{7}$ The impairments in the endogenous antioxidative system lead to
Submission Date: 15-09-2020; Revision Date: 21-10-2020; Accepted Date: 15-11-2020

DOI: 10.5530/ijper.54.4.173 Correspondence: Afzal Haq Asif Department of Pharmacy Practice, College of Clinical Pharmacy, King Faisal University, Al-Ahsa, SAUDI ARABIA.

Phone: +966 555891874 E-mail: ahasif@kfu.edu.sa

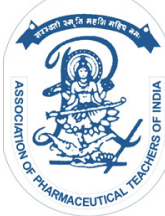

www.ijper.org 
insulin resistance in peripheral metabolic tissues. ${ }^{8}$ Highfat diet consumption alters insulin secretion, glucose utilization, and glucose regulation in skeletal muscles. ${ }^{2}$ Exercise augments insulin-assisted glucose uptake and attenuates hyperinsulinemia and insulin resistance in diabetic rats. ${ }^{9,10}$ Regular intake of saturated fat-rich diet (Western diet) and high intake of vegetable and animal fat leads to insulin resistance ${ }^{11}$ whereas, a diet enriched with polyunsaturated fatty acids could overcome the insulin resistance. ${ }^{12}$ Feeding fish oil to rats has reduced the occurrence of resistance towards insulin, especially in skeletal muscle. ${ }^{13}$ Therefore, dietary intervention and physical exercise are necessary to reduce the incidences of insulin resistance. ${ }^{14}$ Diabetes being a chronic disease requires long-term treatment and therefore, administration of fish oil by oral route will be convenient for the patient. We investigated the effect of lifestyle modification with exercise and fish oil consumption on the occurrence of insulin resistance and fatigue in diabetic rat model developed using combination of high-fat diet and intraperitonial streptozocin.

\section{MATERIALS AND METHODS}

\section{In vivo studies in animals}

Rats (Sprague-Dawley, male, $n=50, n=2$ per cage) were housed in our institutional animal house (Vidya Institute, Bangalore and experiments were performed in accordance with the guidelines of the institutional ethics committee).

\section{High fat dieting, diabetes induction, and glucose monitoring}

The experimental protocol was approved by the Institutional ethics committee and experiments were performed following the guidance from committee members. Sprague Dawley rats $(n=50)$ were equally divided into five groups with 10 animals in each group. Normal control group rats $(n=10)$ were given a regular diet. The rest of the 4 groups $(n=40)$ were on high fat diet for two weeks. Streptozotocin $(35 \mathrm{mg} / \mathrm{kg}$, ip, single dose) was given to these four groups to induce diabetes mellitus. ${ }^{15}$ The glucose level in blood sampled from the tail vein was quantified using AccuCheck (a glucose diagnostic kit - Ser.No.GB 14561388). Rats were designated as diabetic if non-fasting blood sugars were $\geq 250 \mathrm{mg} / \mathrm{dl}$. Before the start of the treatment glucose tolerance test (GTT) was performed. Post 10 weeks of streptozotocin administration (end of the treatment), serum was derived from blood samples, frozen, and analyzed later for biochemical data. Serum glucose concentration was derived using the kits that were available locally (Crest Biosystems, India) via the glucose oxidase peroxidase (GOD-POD) method ${ }^{16}$. Rats with diabetes were divided into four groups as follows: type 2 diabetic control (DC group), diabetic rats with exercise training (DE), diabetic rats with oral dose of fish oil (DF group), diabetic rats with oral dose of fish oil and exercise training (DFE). Fish oil (Marincap capsules $500 \mathrm{mg}$ with -3 fatty acids (35\%), EPA, $18 \%$, and DHA, $12 \% .0 .4 \mathrm{~g} / \mathrm{kg}$ was administered (to group DF and DFE. The lifestyle modification regimen involved for the swimming exercise was $40 \mathrm{~min} /$ day and 5 days/week for groups DE and DFE and oral supplementation of fish oil for group DF and DFE.

\section{Swimming exercise and fatigue test}

The rats from groups DE and DFE were trained to exercise as per the procedure of Rahman $e t$ al. ${ }^{17}$ The swimming chamber was made up of glass $(\mathrm{H}-80 \mathrm{~cm}, \mathrm{~L}-$ $100 \mathrm{~cm}, \mathrm{~W}-80 \mathrm{~cm}$ ) and filled till a height of $60 \mathrm{~cm}$ with warm water $\left(36 \pm 1^{\circ} \mathrm{C}\right)$. The temperature of the water was maintained using a thermostat and air bubbles were produced by using an air pump system. Rats were forced to swim for 40 minutes. The rats from the other groups, NC, DC, and DF were trained alternate days, 10 mins/ session, in the last two weeks of the treatment protocol. Three fatigue tests were conducted. The increase in swimming endurance capacity is when the rats were able to keep their nose and head above the water surface. The rats were forced to swim until exhaustion (signs of fatigue), a point wherein the nose of rats stayed below the surface for $10 \mathrm{sec}$, and swimming time was recorded until exhaustion. ${ }^{18}$

\section{Blood analyses}

\section{Fasting blood glucose (FBG) level, Insulin and Insulin Resistance}

The fasting blood glucose (FBG) levels were determined every 14 days with a blood glucose meter. GT'T was performed by administering a glucose solution $(30 \%$. 0.2 $\mathrm{g} / 100$ gm body weight $)^{19}$ was injected intraperitoneally. ELISA test kis (ALPCO Diagnostics) were used Insulin resistance $=[$ glucose $(\mathrm{mmol} / \mathrm{l}) \times$ Insulin $(\mathrm{IU} / \mathrm{L}) / 22.5$.

\section{Lipid profile}

Blood from 12-hr-fasted rats was collected from the caudal vena cava immediately following sacrifice. The serum levels of total cholesterol (TC), triglyceride (TG), HDL, LDL, and free fatty acid (FAA) were determined. The Friedewald formula was used to estimate VLDL. $\mathrm{VLDL}=\mathrm{TG} / 5 .{ }^{20}$ ELISA kits were used to measure the plasma leptin levels. 


\section{Detection of Oxidative Stress}

Thiobarbituric acid reactive substances (TBARS) levels in plasma

The plasma TBARS level was determined based on the reaction with thiobarbituric acid (TBA) at 90 $-100^{\circ} \mathrm{C}$ in acidic condition $\mathrm{pH} 2-3 .{ }^{21}$ Lipid oxidation materials react with TBA react together and result in the formation of a pink-colored complex which is analyzed spectrophotometrically at $532 \mathrm{~nm}$.

Superoxide dismutase (SOD) and Glutathioneperoxidase activity in Plasma SOD

Total SOD activity was determined according to the method of Sun et al. ${ }^{22}$ The test was performed on the ethanolic fraction of plasma after mixing with ethanol / chloroform mixture (5:3). Plasma GSH-Px (EC 1.6.4.2) activity was measured by an enzymatic reaction leading to a color change, that was monitored spectrophotometrically at $340 \mathrm{~nm}(\mathrm{U} / \mathrm{ml}) .{ }^{23}$

\section{Glycogen content}

The tissue sample of about $1 \mathrm{~g}$ of animal tissue was taken in a centrifuge tube containing $3 \mathrm{ml}$ of $\mathrm{KOH}$ $(30 \%)$ solution. The glycogen was precipitated with the addition of saturated sodium sulfate and ethanol (95\%). The precipitated glycogen was purified and hydrolyzed by adding $0.6 \mathrm{~N} \mathrm{HCl}$ and neutralized the acid by adding sodium hydroxide. Finally, the glucose in the hydrolyzate was determined as reducing sugar. ${ }^{24}$

\section{Statistical analysis}

The Prism 8.0.2 software (GraphPad Software Inc., San Diego, CA, USA) was used for the statistical analysis of the data. All the data were expressed as mean \pm standard error of the mean (SEM). The student's $t$-test was used as appropriate for discrimination. The data were first tested for normality and homogeneity and analyzed using a one-way analysis of variance (ANOVA), followed by Tukey's multiple comparison test for the within-group analysis, given the $5 \%$ significance level.

\section{RESULTS}

\section{Effect of high fat diet and diabetes induction on body-weight}

The initial weight of rats was $220.8 \pm 12 \mathrm{~g}$ and after 2 weeks of treatment with a high-fat diet, the rats gained weight up to $340 \pm 15 \mathrm{~g}$ showing a weight gain of $54.5 \%$. This was significantly higher than the rats fed with a normal diet $(227.1 \pm 10 \mathrm{~g} p<0.001)$.

\section{Glucose tolerance test GTT}

The GTT was performed at the end of the $2^{\text {nd }}$ week to analyze the state of glucose intolerance/insulin resistance of the rats. The GTT showed an increase in glycemia in diabetic control compared to normal control (Figure 1). Post two weeks of the high-fat diet, a single sub-diabetogenic dose of STZ $(35 \mathrm{mg} / \mathrm{kg})$ induced clinical diabetes with hyperglycemia $(<400 \mathrm{mg} / \mathrm{dl})$. Rats with blood glucose levels $>250$ and $<350 \mathrm{mg} / \mathrm{dl}$ were used in the study.

\section{Impact of exercise, consumption of fish oil and dual combination intervention on exercise performance (Endurance capacity)}

The NC and DC groups showed a swimming time of $111 \pm 6$ and $53 \pm 3$ min respectively. When the DC group swimming time was decreased by $52 \%$ indicative of a decline in endurance capacity in diabetic rats. The diabetic rats were significantly exhausted at a much quicker. However, this fatigue was alleviated significantly in diabetic rats that were subjected to supplementation of fish oil and exercise.

The swimming time in DE, DF, and DFE groups was $96 \pm 5,75 \pm 6$, and $105 \pm 5$ min respectively. When compared with the DC group, there was a significant increase in the swimming time in $\mathrm{DE}(81.1 \%), \mathrm{DF}$ (41.5\%), and DFE (98.1\%) groups (Figure $2 \mathrm{~A}$ ).

The swimming time also increased by $40 \%$ in the DFE group compared to the DF group. Diabetic rats supplemented with fish oil and subjected to exercise training (DFE group) exhibited an increase in endurance capacity than their counterpart groups such as DE $(p<0.001)$ and DF $(p<0.001)$ rats. On the other hand, diabetic rats treated with fish oil alone did not seem to be benefitted from the early recovery from fatigue. However, a noteworthy $49.44 \%$ increase in the endurance time in the DFE group compared to $\mathrm{DE}$ rats, indicates a higher endurance capacity when fish oil was added to exercise training (Figure 2B).

\section{Impact of exercise, consumption of fish oil, and dual combination intervention on FBG levels and insulin related parameters}

Consumption of a high-fat diet for 2 weeks significantly increased the FBG level. When the same animals were injected with STZ, we observed a drastic surge in FBG levels in DC, DF, DE, and DFE. These levels increased gradually until the end of the treatment period and displayed a 2.55 fold increase than the control group but there was a gradual decrease in glucose levels in the groups subjected to exercise and fish oil 
supplementation. The glucose levels lowered significantly in DE (20\%), DF (47\%), and DFE (56\%) groups when compared to untreated diabetic rats (Figure 3).

Insulin levels were altered significantly when compared to the NC group. The combined action of fish oil and exercise in the DFE group has ameliorated this significantly altered insulin level towards normal value (Table 1).

\section{Impact of exercise, consumption of fish oil and dual combination intervention on plasma lipid profile and leptin levels}

The untreated diabetic rats (DC) had shown a remarkable abnormal lipid profile in terms of increased total cholesterol (Figure 4A), triglycerides (Figure 4B), LDL (Figure 4D) and VLDL (Figure 4E), free fatty acid (Figure 4F), leptin (Figure 4G), and decreased HDL (Figure 4C) in comparison with the normal control (NC) group. However, the diabetic rats treated with the combination of fish oil with exercise (DFE) had set back these abnormal values toward normalcy (Figure 4). The effects observed in the DFE group were significantly different than the individual treatment groups, namely, exercise-trained DE or fish oil-fed, DF.
Impact of exercise, consumption of fish oil, and dual combination intervention on antioxidant activity

There was a significant difference in antioxidant levels when compared to the control group. However, the exercise impact on fish oil supplementation in rats with diabetes had brought back the levels of antioxidants towards normalcy (Table 2).

Impact of exercise, consumption of fish oil, and dual combination intervention on glycogen content

The increase in insulin sensitivity in tissues, especially skeletal muscle indicates their capacity to store glucose as glycogen. The glycogen content in skeletal muscle and liver was analyzed. An increase in glycogen content in all treated groups but the DE group did not show a significant effect when compared to disease control (Table 3).

\section{DISCUSSION}

Westernized fat-rich diet with high consumption of soft drinks containing fructose), contributes to the development of metabolic complications. ${ }^{25}$ The

Table 1: Effect of exercise and fish oil supplementation on insulin and insulin resistance in type-2
diabetes mellitus rats.

\begin{tabular}{|c|c|c|c|c|c|}
\hline \multirow{2}{*}{ Parameters } & \multicolumn{5}{|c|}{ Groups } \\
\hline & NC & DC & DE & DF & DFE \\
\hline $\operatorname{MDA}(\mathrm{mmol} / \mathrm{L})$ & $0.95 \pm 0.02$ & $2.42 \pm 0.12^{\star * *}$ & $2.06 \pm 0.03^{* * *}$ & $2.12 \pm 0.06^{* * *}, \#$ & 1.24士0.03*”\#\#,\$\$\$,@@@ \\
\hline SOD (IU/L) & $164.2 \pm 2.2$ & $78.6 \pm 8.6^{* * *}$ & $98.4 \pm 3.4^{* \star *}$ & $104.6 \pm 1.2^{* * *}$ & 128.2士4.2 \\
\hline $\mathrm{GSH}(\mathrm{ng} / \mathrm{mL})$ & $2.02 \pm 0.22$ & $0.48 \pm 0.12^{* \star *}$ & $1.32 \pm 0.16$ & $1.25 \pm 0.19^{*}, \# \#$ & 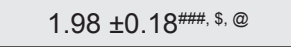 \\
\hline \multicolumn{6}{|c|}{ 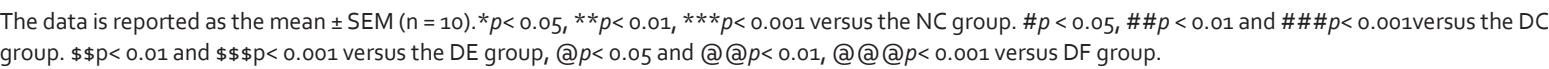 } \\
\hline
\end{tabular}

\begin{tabular}{|c|c|c|c|c|c|}
\hline \multirow{2}{*}{$\begin{array}{c}\text { Glycogen } \\
\text { (mg/gm) }\end{array}$} & \multicolumn{5}{|c|}{ Groups } \\
\hline & NC & DC & DE & DF & DFE \\
\hline Skeletal & $9.13 \pm 1.18$ & $3.36 \pm 1.24^{* *}$ & $4.92 \pm 1.08$ & $7.12 \pm 1.16^{\#}$ & $7.86 \pm 1.61^{\#}$ \\
\hline Liver & $54.22 \pm 4.66$ & $28.36 \pm 5.32^{* * *}$ & $38.35 \pm 2.54$ & $42.14 \pm 3.45^{\#}$ & $49.12 \pm 2.18^{\prime \prime}$ \\
\hline
\end{tabular}




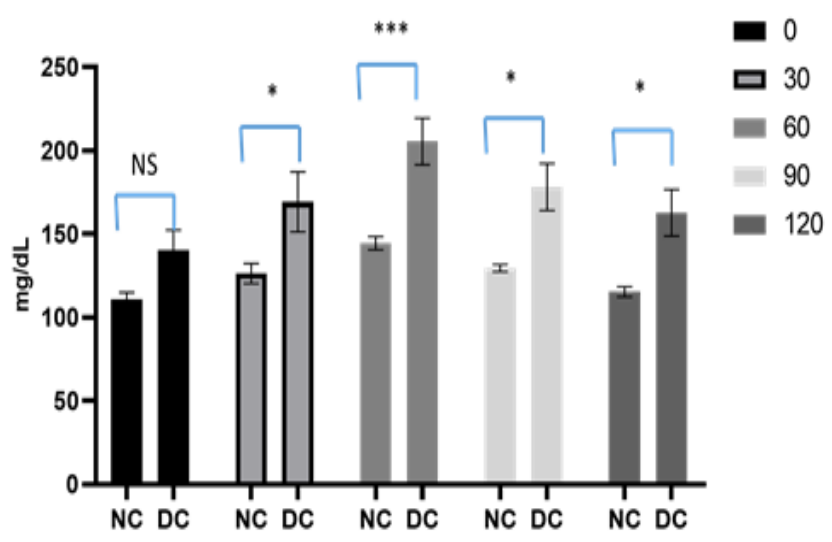

Figure 1. The GTT depicting the effect of $0.2 \mathrm{~g} / 100 \mathrm{gram}$ ip, glucose $(30 \%)$ on blood glucose levels in normal control (NC), and high-fat diet-fed diabetic rats (DC) at 0, 30, 60, 90, and 120 min after glucose administration. Values are means ( \pm SEM) of six animals. Comparison with NC group ${ }^{*} p<0.05,{ }^{* * *} p<0.001$ (Unpaired Student's $t$-test).
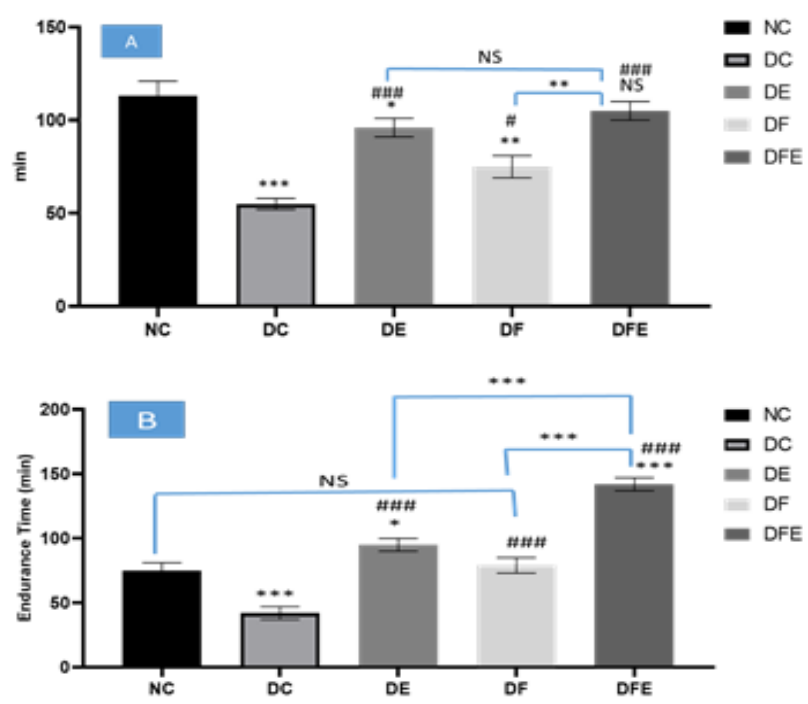

Figure 2. (A) exercise performance and (B) fatigue in diabetes mellitus rats. NC, normal control; DC, diabetes control group;

$D E$, diabetic rats with exercise training; $D E$, the diabetic rat with fish oil supplementation; DFE, the diabetic rat with exercise and fish oil supplementation group. The data is reported as the mean \pm SEM $(n=6)$. * $p<0.05 ;{ }^{* *} p<0.01$; ${ }^{* *} p<$ 0.001 versus the NC group. $\# p<0.05$; and $\# \# \# p<0.001$ versus the DC group.

sedentary lifestyle plays a vital role in making the disease epidemic. Rats fed with a modified diet, i.e. both high fat and high fructose in their diet renders the rats with an increase in weight, insulin resistance, hyperglycemia, hypercholesterolemia, and hypertriglyceridemia with a compensatory increase in insulin levels, a condition similar to insulin resistance state. Our study demonstrates that, in insulin-resistant diabetic rats, combination intervention of physical exercise and a daily dose of fish oil supplementation improves insulin sensitivity and relieves fatigue. The advantage of swimming as an exercise or diets enriched with polyphenols on

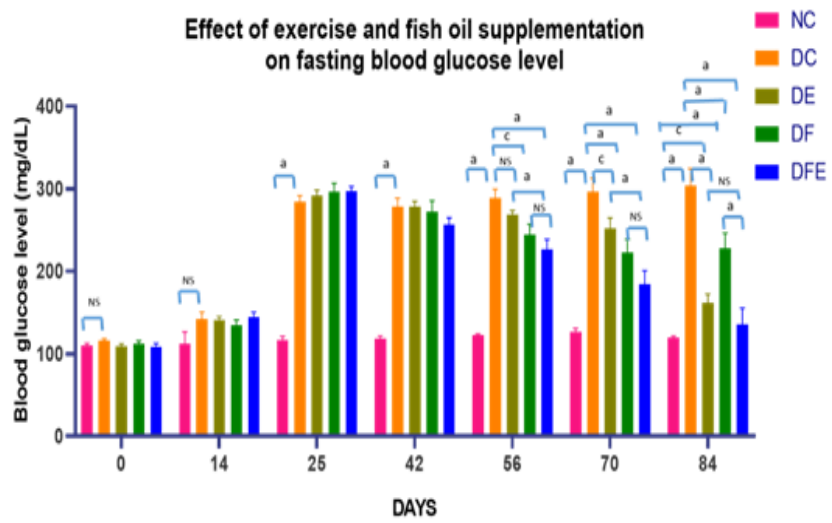

Figure 3. Fasting blood glucose levels. NC: normal control; DC: diabetes control group; DE: diabetic rat with exercise training; DF: diabetic rat with fish oil supplementation; DFE: diabetic rat with exercise training and fish oil supplementation. The values are reported as the mean \pm SEM $(n=10) . c p<0.05 ;{ }^{b} p<0.01 ;{ }^{a} p<0.001$. NS = Not significant.
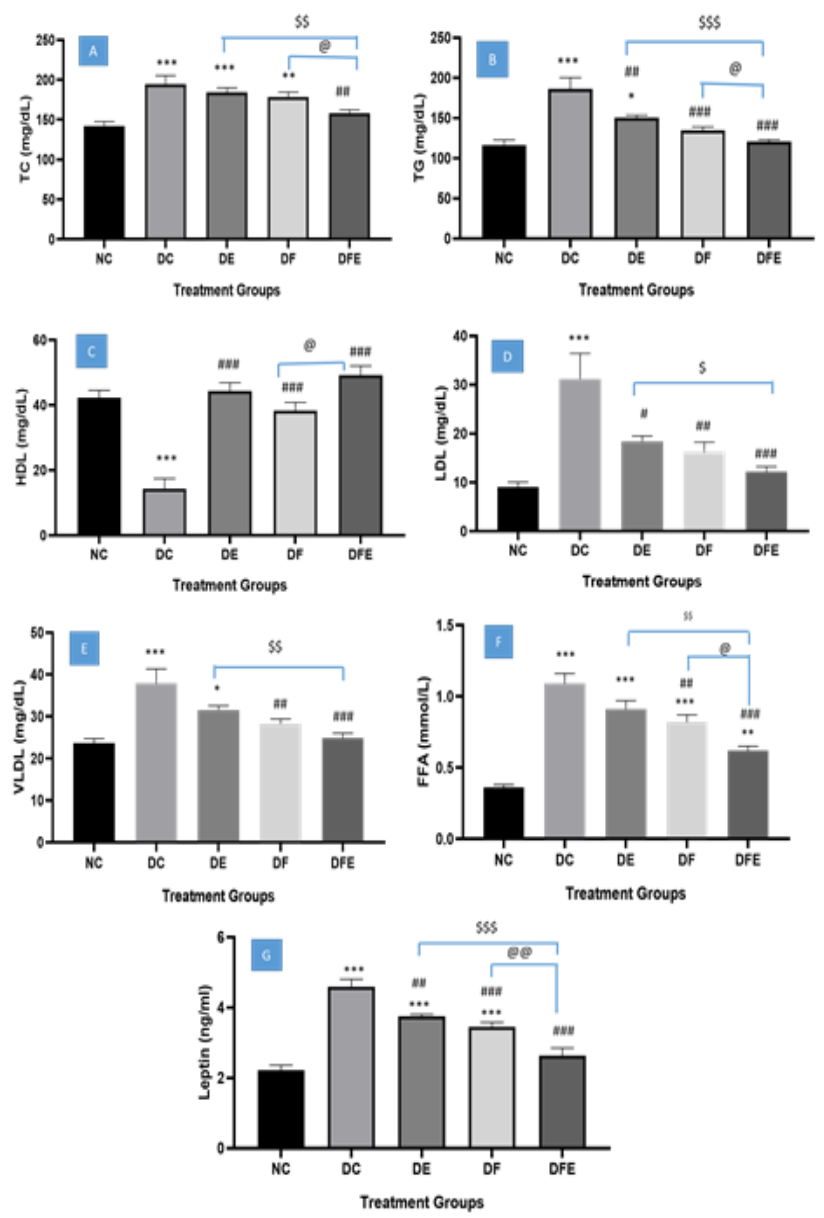

Figure 4. Impact of exercise, consumption of fish oil, and dual combination intervention on plasma A: Total Cholesterol (TC), B: Triglycerides (TG), C: High-density lipoprotein (HDL), D: Low-density lipoprotein (LDL), E: Very low-density lipoprotein (VLDL), F: Free fatty acid (FAA) and G: Leptin]. The data is reported as the mean $\pm \operatorname{SEM}(n=10) .{ }^{*} p<0.05,{ }^{* *} p<0.01$, ${ }^{* * *} p$

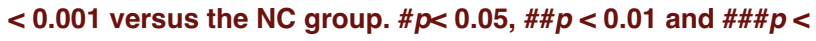
0.001 versus the $D C$ group. $\$ \$ p<0.01$ and $\$ \$ \$ p<0.001$ versus the DE group, @ $p<0.05$ and @@ $p<0.01$ versus DF group. 
physiologic parameters in metabolic syndrome has been studied enormously, but none have studied the combination in rats.

In the past, the ability of polyphenol to prevent the development of diet-induced metabolic alterations was studied as a prophylactic, as the rats were treated with polyphenols before disease induction. In the present study, a combination of fish oil supplementation and swimming was used after the development of insulin resistance to understand the curative potential of fish oil and as a dual strategy for life-style modification. Nutritional supplementation of fish oil was sufficient to decrease rats' body weight gain by $6 \%$. The incidence of diabetes mellitus reduces drastically with an even 5\% decrease in body weight. ${ }^{26,27}$ Additionally, our study revealed the prevention of weight gain and promoting overall metabolic improvement with a dual lifestyle intervention with exercise and diet. Such prevention of weight gain is not observed in humans on either polyphenol intake or exercise.

The reduced glucose uptake and utilization (insulin resistance) can be explained by the fatty acid-glucose cycle, wherein the hyperglycemia state had occurred due to an increase in FFA. ${ }^{28}$ The untreated diabetic rats (DC) showed a significant increase in free fatty acid, TC, TG, LDL, and VLDL ( Figure 4) but the combination of fish oil supplementation and exercise (DFE), resulted in lower insulinemia and insulin resistance compared to the untreated rats of the diabetic group (DC), suggesting an improvement in insulin sensitivity (Table 1). The other possible mechanism in the improvement of insulin sensitivity might be due to an increase in glycogen storage in skeletal muscle. Insulin sensitivity increased in rats belonging to the DFE group because these rats had an increase in glycogen content when compared with other treated groups.

Visceral adiposity causes an increase in insulin as well as leptin levels and severe metabolic consequences. ${ }^{29,30}$ This demonstrates that these untreated diabetic rats had unfavorable metabolic disturbances, like intolerance in glucose during a GTT test, insulin resistance, leptin resistance, and hyperlipidemia. The synergistic effect of swimming exercise on fish oil supplementation that helped to normalize the metabolic abnormalities such as serum TC, TG, HDL, LDL, VLDL, and leptin. The probable mechanism may be blocking the synthesis of adiponectin that is directly influenced by the concentrations of free fatty acids.

The lipid profile and leptinemia were significantly reduced by the combination treatment of fish oil and exercise, thereby ameliorating the levels of FFA and insulin resistance. Past research ${ }^{31}$ had proven the efficiency of adiponectin in controlling FFA, lipids, and insulin sensitivity. The ameliorating effect of the DFE group on the FFA levels was significant when compared to DE and DF groups and might have increased the insulin sensitivity due to improvement in adiponectin. Improvement in adiponectin levels improves insulin resistance. ${ }^{32}$ The increase in insulin sensitivity in the DFE group correlates with a significant $(49.44 \%)$ increase in the endurance time, indicating the higher exercise capacity when fish oil was administered in addition to exercise training. The current study also demonstrated a significant decrease in FBG level on completion of therapy in the DFE group which indicates a higher peripheral utilization of glucose which correlates with an increase in endurance time. Regular intake of diet (Western diet) rich in fat (saturated) has led to a state of insulin resistance ${ }^{11}$ and on the other hand diet rich in PUFA would have a positive impact on insulin resistance. ${ }^{12}$ Feeding fish oil to rats on diet with high fat has reduced the occurrence of resistance towards insulin, especially in skeletal muscle. ${ }^{13}$

The DFE group also demonstrated the synergistic effect of exercise and fish oil supplementation in reducing the oxidative stress as observed from reduced MDA levels and simultaneously increased the levels of endogenous antioxidant enzymes; SOD and GSH (Table 2). The improved insulin resistance and hyperglycemic state might be the reason for this effect. Oxidative stress associated with diabetes is the leading cause of obesity that further aggravates lipid peroxidation and suppresses antioxidant activities. A high-fat diet is responsible for increasing the burden on the endogenous antioxidative system. ${ }^{7}$ Therefore, the impaired endogenous antioxidative system leads to insulin resistance in peripheral metabolic tissues. ${ }^{8}$ In vivo studies have demonstrated the effect of fish oil as a free radical scavenger. ${ }^{33}$ and Chautan et al. ${ }^{34}$ demonstrated stimulation of vitamin $\mathrm{E}$ incorporation into membranes modulated by Omega 3 fatty acid and thus prevention of lipid peroxidation. Regular controlled exercise maintains a balance between inflammatory and pro-inflammatory cytokines, lowers the leptin levels, and oxidative stress. ${ }^{35}$ The etiology behind the tiredness and fatigue symptoms in diabetes patients is still not very clear. Associated obesity and insulin resistance are the responsible factors for fatigue $e^{36,37}$ and as observed from the swimming exercise test, the dual intervention of physical training and dietary modification with fish oil could overcome the fatigue as observed from in DFE group (Figure 2A and $\mathrm{B})$.

A decrease in glycogen content of untreated diabetic rats were noted (Table 3). DE group showed a tendency 
toward an insignificant increase in glycogen content and significantly raised in DF and DFE groups. Loss of weight was seen in untreated diabetic rats and not in treated groups because these groups have increased the glycogen content in both the tissues and the same would have been utilized for energy expenditure instead of storage, which in turn prevented the loss of weight.

\section{CONCLUSION}

Dietary supplementation of fish oil has could repair the abnormal free radical-induced oxidative changes and reduced lipid peroxidation. Therefore, fatty acids like EPA and DHA are effective ingredients for food functionalization for added therapeutic benefits. Food fortification and dietary supplementation in controlling the disease-induced damage caused by chronic diseases like diabetes resulting from the imbalance between oxidant/antioxidant defense mechanisms would play a significant role. Decreased glycogen content in liver and skeletal tissue in diabetes leading to fatigue was significantly altered when the lifestyle was modified with diet as well as exercise. Moderate physical training along with healthy dietary modifications could reverse the metabolic damage and enhance exercise endurance capacity thus facilitating early recovery from fatigue. Therefore, a combination of moderate physical exercise and incorporation of any source of omega 3 fatty acids like fish oil in the diet would be a new, effective, and powerful treatment modality for the management of chronic diabetic complications.

\section{Author Contributions}

Conceptualization, A.H.A, P.K.N, U.C and R.S.T ; Data curation, A.H.A, P.K.N, G.M.B, N.S and U.C; Formal analysis, G.M.B; Funding acquisition, A.H.A, G.M.B, N.S and R.S.T ; Investigation, A.H.A, P.K.N, G.M.B, N.S, U.C and R.S.T ; Methodology, A.H.A, P.K.N, G.M.B, N.S, U.C and R.S.T ; Project administration, A.H.A, P.K.N, G.M.B, N.S and U.C; Resources, A.H.A, P.K.N, N.S, U.C and R.S.T ; Software, P.K.N and U.C; Supervision, G.M.B, N.S and R.S.T ; Validation, A.H.A, P.K.N, G.M.B, N.S, U.C and R.S.T ; Writing - original draft, A.H.A, P.K.N, G.M.B, N.S, U.C and R.S.T ; Writing - review \& editing, A.H.A, P.K.N and N.S.

\section{Funding}

The authors extend their appreciation to the Deputyship for Research \& Innovation, Ministry of Education in Saudi Arabia for funding this research work through the project number IFT20115.

\section{ACKNOWLEDGEMENT}

The authors thank the Deanship of Scientific Research, King Faisal University, Al-Ahsa, Saudi Arabia for their support.

\section{CONFLICT OF INTEREST}

The authors declare no conflicts of interest.

\section{ABBREVIATIONS}

GTT: Glucose Tolerance Test; GOD-POD: Glucose Oxidase Peroxidase; DC group: Type 2 Diabetic Control; DFE: Oral Dose Of Fish Oil And Exercise Training; FBG: Fasting Blood Glucose; TC: Total Cholesterol; TG: Triglyceride; TBARS: Thiobarbituric Acid Reactive Substances; SOD: Superoxide Dismutase; TBA: Thiobarbituric Acid.

\section{REFERENCES}

1. Hu FB, Manson JE, Stampfer MJ, Colditz G, Liu S, Solomon CG, et al. Diet, lifestyle, and the risk of type 2 diabetes mellitus in women New England. Journal of Medicine. 2001;345(11):790-7.

2. Lasselin J, Layé S, Dexpert S, Aubert A, Gonzalez C, Gin H, et al. Fatigue symptoms relate to systemic inflammation in patients with type 2 diabete. Brain, Behavior and Immunity. 2012;26(8):1211-9.

3. Wright DW, Hansen R, Mondon C, Reaven G. Sucrose-induced insulin resistance in the rat: Modulation by exercise and diet. The American Journal of Clinical Nutrition. 1983;38(6):879-83.

4. Blachnio-Zabielska AU, Chacinska M, Vendelbo MH, Zabielski P. The crucial role of $\mathrm{C} 18-\mathrm{Cer}$ in fat-induced skeletal muscle insulin resistance. Cellular Physiology and Biochemistry. 2016;40(5):1207-20.

5. Calder PC. The relationship between the fatty acid composition of immune cells and their function. Prostaglandins, Leukotrienes and Essential Fatty Acids. 2008;79(3-5):101-8.

6. Hurrle S, Hsu WH. The etiology of oxidative stress in insulin resistance. Biomedical Journal. 2017;40(5):257-62.

7. Saha SK, Lee SB, Won J, Choi HY, Kim K, Yang GM, et al. Correlation between Oxidative Stress Nutrition and Cancer Initiation. International Journal of Molecular Sciences. 2017;18(7):1544.

8. Turcotte LP, Fisher JS. Skeletal muscle insulin resistance: Roles of fatty acid metabolism and exercise. Physical Therapy. 2008;88(11):1279-96.

9. Roberts CK, Hevener AL, Barnard RJ. Metabolic syndrome and insulin resistance: underlying causes and modification by exercise training. Comprehensive Physiology. 2013;3(1):1-58. https://doi.org/10.1002/cphy. c110062.

10. Andrade EF, Silva VO, Moura NO, Foureaux RC, Orlando DR, Moura RF, et al. Physical Exercise Improves Glycemic and Inflammatory Profile and Attenuates Progression of Periodontitis in Diabetic Rats (HFD/STZ). Nutrients. 2018;10(11):1702.

11. Pedersen $\mathrm{O}$, Kahn CR, Flier JS, Kahn BB. High fat feeding causes insulin resistance and a marked decrease in the expression of glucose transporters (Glut 4) in fat cells of rats. Endocrinology. 1991;129(2):771-7.

12. Yki-Järvinen H. Nutritional Modulation of Non-Alcoholic Fatty Liver Disease and Insulin Resistance. Nutrients. 2015;7(11):9127-38.

13. Lepretti M, Martucciello S, Burgos AMA, Putti R, Lionetti L. Omega-3 Fatty Acids and Insulin Resistance: Focus on the Regulation of Mitochondria and Endoplasmic Reticulum Stress Nutrients. 2018;10(3):350. https://doi. org/10.3390/nu10030350. 
14. Santeusanio F, DiLoreto C, Lucidi P, Murdolo G, DeCicco A, Parlanti N, et al. Diabetes and exercise. Journal of Endocrinological Investigation. 2003;26(9):937-40.

15. Srinivasan K, Viswanad B, Asrat L, Kaul C, Ramarao P. Combination of high-fat diet-fed and low-dose streptozotocin-treated rat: A model for type 2 diabetes and pharmacological screening. Pharmacol Res. 2005;52(4):31320.

16. Kinoshita T, Hiraga Y, Nakamura N, Kitajo A, linuma F. Determination of glucose in blood using glucose oxidase-peroxidase system and 8-hydroxyquinoline-panisidine. Chem Pharm Bull. 1979;27(2):568-70.

17. Rahman MM, Lee SJ, Mun AR, Adam GO, Park RM, Kim GB, et al. Relationships between blood $\mathrm{Mg} 2+$ and energy metabolites/enzymes after acute exhaustive swimming exercise in rats. Biol Trace Elem Res. 2014;161(1):85-90.

18. Liu Y, Zhang HG, Li XH. A Chinese herbal decoction Danggui Buxue Tang improves chronic fatigue syndrome induced by food restriction and forced swimming in rats. Phytother Res. 2011;25(12):1825-32.

19. Jørgensen MS, Tornqvist KS, Hvid H. Calculation of Glucose Dose for Intraperitoneal Glucose Tolerance Tests in Lean and Obese Mice. Journal of the American Association for Laboratory Animal Science. 2017;56(1):95-7.

20. Friedewald WT, Levy RI, Fredrickson DS. Estimation of the concentration of low-density lipoprotein cholesterol in plasma without use of the preparative ultracentrifuge. Clin Chem. 1972;18(6):499-502.

21. Esterbauer $\mathrm{H}$, Cheeseman $\mathrm{KH}$. Determination of aldehydic lipid peroxidation products: malonaldehyde and 4-hydroxynonenal. In Methods in Enzymology: Elsevier. 1990;186:407-21.

22. Sun Y, Oberley LW, Li Y. A simple method for clinical assay of superoxide dismutase. Clin Chem 1988;34(3):497-500.

23. Paglia DE, Valentine WN. Studies on the quantitative and qualitative characterization of erythrocyte glutathione peroxidase. J Lab Clin Med. 1967;70(1):158-69.

24. Hassid W, Abraham S. Chemical procedures for analysis of polysaccharides. 1957.

25. Astrup A, Finer N. Redefining type 2 diabetes:'diabesity'or 'obesity dependent diabetes mellitus'?. Obesity Reviews. 2000;1(2):57-9.

26. Tuomilehto J, Lindström J, Eriksson JG, Valle TT, Hämäläinen H, llanneParikka $\mathrm{P}$, et al. Prevention of type 2 diabetes mellitus by changes in lifestyle

among subjects with impaired glucose tolerance. New England Journal of Medicine. 2001;344(18):1343-50.

27. Knowler WC, Barrett-Connor E, Fowler SE, Hamman RF, Lachin JM, Walker EA, et al. Diabetes Prevention Program Research Group Reduction in the incidence of type 2 diabetes with lifestyle intervention or metformin. $\mathrm{N}$ Engl J Med. 2002;346(6):393-403.

28. Randle P. The glucose fatty-acid cyclelts role in insulin sensitivity and the metabolic disturbances of diabetes mellitus. Lancet. 1963;1:785-9.

29. Wolden-Hanson T, Mitton D, McCants R, Yellon S, Wilkinson C, Matsumoto A, et al. Daily melatonin administration to middle-aged male rats suppresses body weight intraabdominal adiposity and plasma leptin and insulin independent of food intake and total body fat. Endocrinology. 2000;141(2):487-97.

30. Ríos-Lugo MJ, Cano P, Jiménez-Ortega V, Fernández-Mateos MP, Scacchi PA, Cardinali DP, et al. Melatonin effect on plasma adiponectin leptin insulin, glucose, triglycerides and cholesterol in normal and high fat-fed rats. J Pineal Res. 2010;49(4):342-8.

31. Simpson KA, Singh MAF. Effects of exercise on adiponectin: A systematic review. Obesity. 2008;16(2):241-56.

32. Bi Y, Wang T, Xu M, Xu Y, Li M, Lu J, et al. Advanced research on risk factors of type 2 diabetes. Diabetes/Metabolism Research and Reviews. 2012;28:329.

33. Fisher $\mathrm{M}$, Levine $\mathrm{PH}$. Effects of dietary $\omega 3$ fatty acid supplementation on leukocyte free radical production. In Health Effects of Omega 3 Polyunsaturated Fatty Acids in Sea foods Karger Publishers. 1991;66:245-9.

34. Chautan $\mathrm{M}$, Calaf $\mathrm{R}$, Leonardi J, Charbonnier M, Andre M, Portugal, et al. Inverse modifications of heart and liver alpha-tocopherol status by various dietary $n-6 / n-3$ polyunsaturated fatty acid ratios. J Lipid Res. 1990;31(12):2201-8.

35. Samjoo I, Safdar A, Hamadeh M, Raha S, Tarnopolsky M. The effect of endurance exercise on both skeletal muscle and systemic oxidative stress in previously sedentary obese men. Nutr Diabetes. 2013;3(9):e88.

36. Wang ST, Hwang DF, Chen RH, Chen YC. Effect of deep sea water on the exercise-induced fatigue of rats. J Food Drug Anal. 2009;17(2).

37. Vgontzas AN, Papanicolaou DA, Bixler EO, Hopper K, Lotsikas A, Lin HM, et al. Sleep apnea and daytime sleepiness and fatigue: Relation to visceral obesity, insulin resistance and hypercytokinemia. J Clin Endocrinol Metab. 2000;85(3):1151-8.

\section{PICTORIAL ABSTRACT}

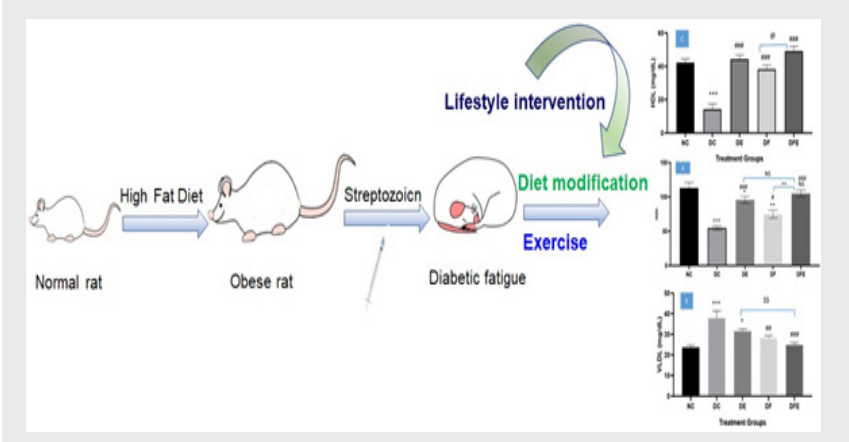

\section{SUMMARY}

Dietary intervention and physical exercise are necessary to reduce the incidences of insulin resistance Diabetes being a chronic disease requires long-term treatment and therefore, administration of fish oil by oral route will be convenient for the patient.

\section{About Authors}

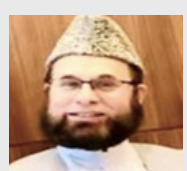

Dr. Afzal completed M.B.B.S. from Nishtar Medical University, Multan, in 1984 and M.Phil. from Postgraduate Medical Institute, University of Punjab, Lahore, Pakistan in Pharmacology in 1995. He is deeply involved in teaching, training, curriculum development and quality management in College of Clinical Pharmacy, King Faisal University, Saudi Arabia since 2005. His area of interest in research is nephrotoxicity of drugs and its protection and internal medicine. He is also working on antidiabetic activity of indigenous plants. He is member of research group working on development of novel pharmaceutical formulations of various drugs. He has about 40 publications on is credit.

Cite this article: Asif AH, Nanjundan PK, Basavarajappa GM, Sreeharsha N, Chandur U, Roopashree TS. Insulin Resistance Modulation with Lifestyle Modification- Proof-of-Concept Study in Rats. Indian J of Pharmaceutical Education and Research. 2020;54(4):999-1006. 